\title{
Identification of Ventricular Tachycardia Using Intracavitary Ventricular Electrograms: Analysis of Time and Frequency Domain Patterns
}

\author{
DONGPING LIN, LORENZO A. DICARLO,** and JANICE M. JENKINS
}

From the Department of Electrical Engineering and Computer Science, School of Engineering; *St. Joseph Mercy Hospital of the Catherine McAuley Health Center and the School of Medicine, University of Michigan, Ann Arbor, MI USA

\begin{abstract}
LIN, D., ET AL.: Identification of ventricular tachycardia using intracavitary ventricular electrograms: analysis of time and frequency domain patterns. Tachycardia detection by implantable antitachycardia devices using rate alone has major limitations. Several alternative methods have been proposed to distinguish ventricular tachycardia or ventricular fibrillation from normal sinus rhythm using intracardiac electrograms. These methods have not been tested, however, for recognition of ventricular tachycardia in patients with abnormal surface QRS conduction during sinus rhythm or with antiarrhythmic drug therapy. In this study, three techniques for the indentification of ventricular tachycardia from intracavitary bipolar ventricular electrograms were examined and compared: correlation waveform analysis, amplitude distribution analysis, and spectral analysis using Fast Fourier transformation. Thirty episodes of induced monomorphic ventricular tachycardia were analyzed and compared sinus rhythm in four groups of patients with: I. Normal surface QRS conduction during sinus rhythm without antiarrhythmic drug therapy (five episodes); II. Intraventricular conduction delay or bundle branch block during sinus rhythm without antiarrhythmic drug therapy (nine episodes); III. Normal surface QRS conduction during sinus rhythm with antiarrhythmic therapy (six episodes); and IV. Intraventricular conduction delay or bundle branch block during sinus rhythm with antiarrhythmic drug therapy (ten episodes). Correlation waveform analysis had $100 \%$ sensitivity and specificity in distinguishing ventricular tachycardia from sinus rhythm, even in the presence of an intraventricular conduction delay, bundle branch block, and antiarrhythmic drug therapy. In contrast, amplitude distribution analysis differentiated $15 / 30$ episodes (50.0\%) of ventricular tachycardia from sinus rhythm, and a maximum of $18 / 30$ episodes (60.0\%) of ventricular tachycardia were identified by specal analysis using Fast Fourier transformation. Correlation waveform analysis appears to be a reliable technique to discriminate ventricular tachycardia from sinus rhythm using intracavitary ventricular electrograms. Its computational demands are modest, making it suitable for consideration in an implantable antitachycardia device. (PACE, Vol. 11, November 1988)
\end{abstract}

ventricular tachycardia, computer analysis, ventricular electrograms, waveform analysis, morphology

This work was partially supported by a grant from the Whitaker Foundation, NSF grants No. ECS-8351215 and ECS-8506494, NIH grant RO1 HL35554 and a grant from the Vice President for Research, University of Michigan.

Address for reprints: Lorenzo A. DiCarlo, M.D., Reichert Health Building R-3003, Catherine McAuley Health Center, P.O. Box 994, Ann Arbor, MI 48106, U.S.A.

Received April 12, 1988; accepted June 8, 1988.

\section{Introduction}

The efficacy of antitachycardia devices for the treatment of ventricular tachycardia refractory to antiarrhythmic medication depends upon the reliable identification of ventricular tachycardia. Methods proposed for this purpose have included dection of an abrupt increase in heart 
rate, ${ }^{1.2}$ determination of a tachycardia rate and duration which exceed an arbitrarily defined minimum, ${ }^{1-5}$ and observation of a stable RR interval during the tachycardia. ${ }^{6}$ Additional methods suggested for determining the site of tachycardia origin have utilized differences in the intracavitary ventricular electrograms recorded during sinus rhythm and ventricular tachycardia from bipolar electrode leads positioned in the right ventricular apex. These latter methods have considered the ventricular electrogram amplitude and slew rate, ${ }^{7}$ gradient pattern analysis, ${ }^{8}$ power spectrum analysis using Fast Fourier transformation, ${ }^{9}$ probability density function, ${ }^{10}$ the area of difference between waveforms recorded during sinus rhythm and ventricular tachycardia, ${ }^{11}$ electrogram morphology, ${ }^{12}$ and multiple electrodes. ${ }^{13}$

While some of these methods have been useful in detecting the occurrence of ventricular tachycardia in patients having a normal electrocardiographic QRS complex during sinus rhythm, they have not been uniformly reliable. ${ }^{14,15}$ Their reliability has not yet been tested in patients with a wide-QRS during sinus rhythm due to an intraventricular conduction delay or a bundle branch block. The impact of antiarrhythmic drug therapy upon electrogram analysis for the detection of ventricular tachycardia has also not been determined.

In this study, we evaluated a new method for identifying ventricular tachycardia from intracavitary, bipolar ventricular electrograms using a correlation method of waveform analysis. A similar method has been traditionally applied to waveform analysis of surface electrocardiograms. ${ }^{16-20}$ We also compared its reliability to that of amplitude distribution analysis ${ }^{20}$ and power spectrum analysis in identifying ventricular tachycardia in the presence or absence of antiarrhythmic drug therapy in patients having either a normal surface electrocardiogram QRS, an intraventricular conduction delay, or bundle branch block during sinus rhythm.

\section{Methods}

\section{Patient Population}

Electrograms analyzed were recorded during clinical cardiac electrophysiology studies of con- secutive patients undergaing evaluation and treatment for documented episodes of sustained ventricular tachycardia having a duration of at least 30 seconds or causing hemodynamic collapse. There were 13 men and 5 women of ages 14 to 83 years. There was clinical evidence of structural heart disease in 17 patients: 15 patients had ischemic cardiomyopathy, one patient had a congestive cardiomyopathy due to hypertension, and one patient had valvular heart disease. One patient had no clinical evidence of heart disease.

\section{Electrophysiology Study}

Patients were studied in a fasting postabsorbtive state after sedation with 1-3 mg of intravenous medazolam. After administering $1 \%$ lidocaine for local anesthesia, three 7 French sidearm sheaths (Cordis Corporation, Miami, FL, USA) were positioned in the right femoral vein using the Seldinger technique. During baseline studies without antiarrhythmic drug therapy, three 6 French quadripolar electrode catheters with an interelectrode distance of $1 \mathrm{~cm}$ (USCI di vision, C.R. Bard Inc. Billerica, MA, USA) were introduced and advanced under fluoroscopic guidance to the high right atrium, tricuspid valve (for His bundle recording), and right ventricular apex respectively. After completion of atrial programmed stimulation, the right atrial electrode catheter was securely positioned in the right ventricular apex and remained fixed in this position for the duration of the study. Ventricular electrograms were recorded from this catheter during sinus rhythm, programmed ventricular stimulation, and induced ventricular tachycardia. Sustained ventricular tachycardia was induced using a previously described protocol for programmed ventricular stimulation. ${ }^{21}$ During subsequent electropharmacological testing of antiarrhythmic drugs, two quadripolar electrode catheters were positioned in the right ventricular apex with one catheter being used only for the recording of ventricular electrograms during sinus rhythm and induced ventricular tachycardia.

\section{Data Acquisition}

All recordings were made with the patients lying supine. Immediately before programmed ventricular stimulation, a 12-lead electrocardio- 
gram was recorded during sinus rhythm. Ventricular electrograms were simultaneously recorded on FM tape (Hewlett Packard 3968A and 3964A San Diego, CA, USA) from the distal pair of electrodes of the right ventricular apex recording catheter using amplifiers (Siemens Mingograf-7 Solona, Sweden) with a filter setting of 1 to 500 Hz. Tape Speed was $3 \frac{3}{4}$ inches per second with a bandwidth of $0-1250 \mathrm{~Hz}$. The recorded ventricular electrograms were subsequently digitized on an IBM PC/XT with Tecmar Lab Master (Scientific Solutions, Inc.) analog-to-digital system at a sampling frequency of 1000 Hertz (sample interval equals one millisecond) during playback of the tapes. Tracings of the surface lead electrocardiogram and ventricular electrogram were also obtained during playback with Gould (Brush 220) strip chart recorders. The programs for digitization and signal analysis were written in $C$ and assembly language.

\section{Methods of Analysis}

The surface 12-lead electrocardiographic recordings during sinus rhythm were examined for the presence of intraventricular conduction delay, right bundle branch block, or left bundle branch block, defined as having a QRS duration $\geq 0.12$ seconds and a morphology conforming to the criteria of Marriott. ${ }^{22}$ Paired data sets, consisting of 10-second passages of both sinus rhythm and ventricular tachycardia from the same patient, were digitized for computer analysis. There were five paired data sets (sinus rhythm vs ventricular tachycardia) from five patients with a normal QRS during sinus rhythm and no antiarrhythmic drug therapy (Group I), nine paired data sets from eight patients with an intraventricular condution delay or bundle branch block during sinus rhythm without antiarrhythmic drug therapy (Group II), six paired data sets from five patients with a normal QRS during sinus rhythm with antiarrhythmic drug therapy (Group III), and ten paired data sets from eight patients with an intraventricular conduction delay or bundle branch block during sinus rhythm with antiarrhythmic drug therapy (Group IV).

Three digital signal processing methods were applied to each paired data set to determine whether ventricular tachycardia could be distinguished from sinus rhythm: (1) correlation wave- form analysis, (2) amplitude distribution analysis, and (3) power spectrum analysis.

\section{Correlation Waveform Analysis}

A statistical measure of the difference between two curves was applied for correlation waveform analysis. A standard equation for the correlation coefficient ${ }^{23}$ was used to detect morphological changes in the intracavitary ventricular electrograms during ventricular tachycardia when compared to the electrograms during sinus rhythm.

A template of the ventricular electrograms during sinus rhythm was obtained by signal averaging the ventricular depolarization waveforms from a passage of sinus rhythm to produce a representative waveform. A window size of 64 milliseconds was selected for the ventricular depolarization resulting in 64 sample points obtained at a 1000 Hertz sampling rate. In a second analysis of the same sinus rhythm data set, ventricular waveforms with a correlation coefficient of $\geq 0.90$ with the representative waveform were retained in the residual average and the remaining waveforms, representing ventricular premature depolarizations during sinus rhythm, were rejected. This revised representative waveform then constituted the sinus rhythm template for comparison of subsequent waveforms during sinus rhythm and ventricular tachycardia. A second, separate passage of sinus rhythm and a passage of ventricular tachycardia were then correlated on a beat-bybeat basis using the sinus rhythm template as the reference.

The following equation was used to calculate the correlation coefficient:

$$
p=\frac{\sum_{i=1}^{M}\left(T_{i}-\mu_{T}\right)\left(X_{i}-\mu_{X}\right)}{\sqrt{\sum_{i=1}^{M}\left(T_{i}-\mu_{T}\right)^{2} \sum_{i=1}^{M}\left(X_{i}-\mu_{X}\right)^{2}}}
$$

Where

$M=$ the window size of the ventricular depolarization

$T_{i}=$ the ith sample point in the sinus rhythm template

$X_{i}=$ the $i$ th sample point of the ventricular depolarization being examined

$\mu_{\mathrm{T}}=$ mean of sinus rhythm template

$\mu_{\mathrm{X}}=$ mean of the ventricular depolarization being examined 


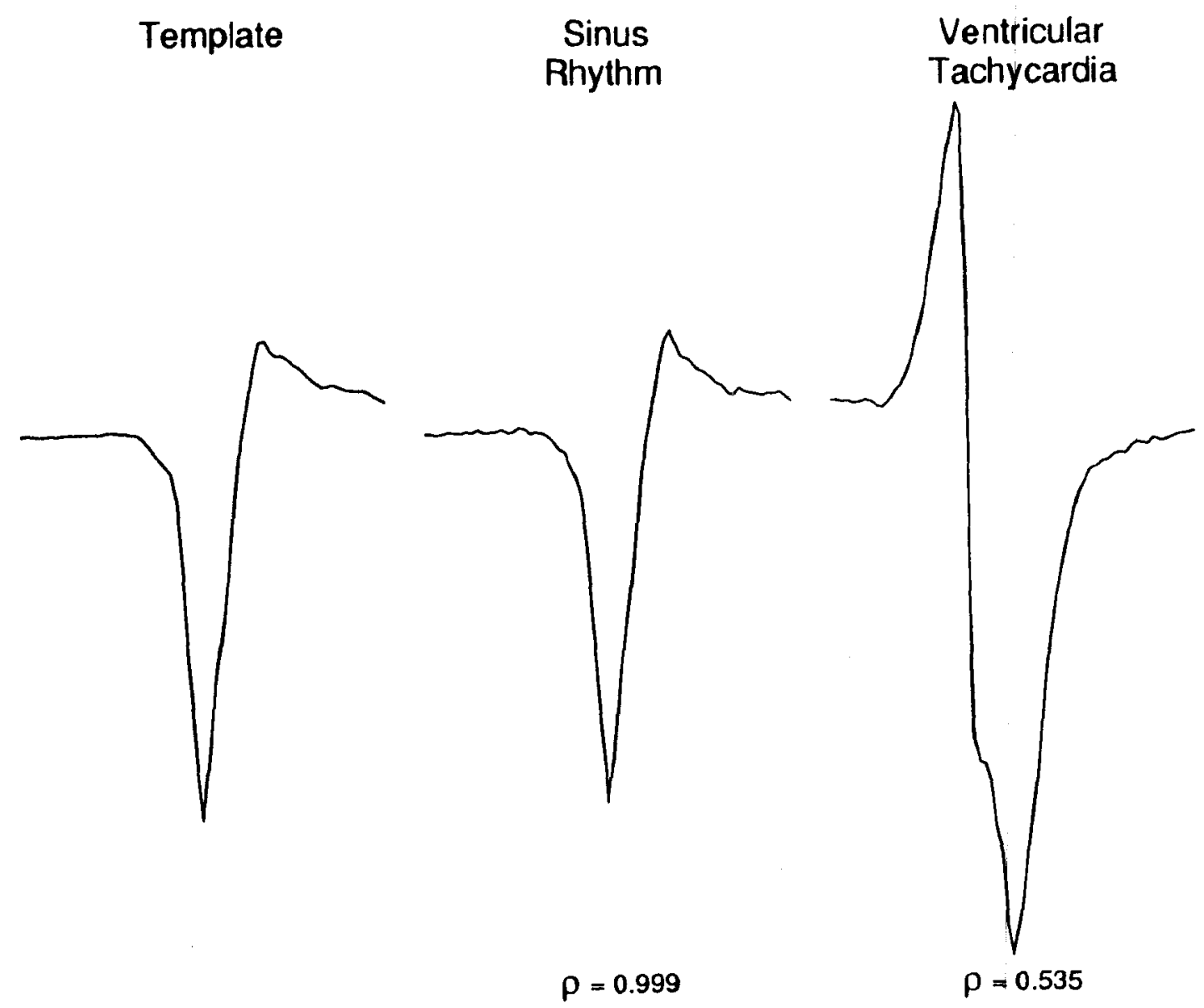

Figure 1. Example of correlation waveform analysis of a ventricular intracavitary electrogram. A template of ventricular depolarization (left) was created from a 10-second passage of signalaveraged ventricular depolarizations. A typical ventricular depolarization during sinus rhythm (center) has a correlation coefficient $(p)>0.90$ while a ventricular depolarization during ventricular tachycardia (right) exhibits a change in morphology with resultant $\mathbf{p}=0.535$. The sampling rate is $1000 \mathrm{Hertz}$ and the window size is 65 milliseconds.

The correlation coefficient $(p)$ represented a measure of similarity in the range $-1<\mathrm{p}<+1$, where +1 indicated a perfect match. Figure 1 shows an example of correlation values of intracavitary ventricular electrograms in sinus rhythm and in ventricular tachycardia, respectively, with the sinus rhythm template. The correlation coefficient was used for discriminating beats in sinus rhythm from beats in ventricular tachycardia.

\section{Amplitude Distribution Analysis}

Amplitude distribution analysis was performed by examining the signal amplitude distri- bution within each cardiac cycle of a passage of intracavitary ventricular electrograms during sinus rhythm and ventricular tachycardia. This is a digital method patterned after a hardware technique developed by Mirowski et al. ${ }^{10}$ for the commercially available automatic implantable cardioverter-defibrillator, and has also been called probability density function. It is based upon the premise that the amount of time which is spent at baseline in each cardiac cycle is significantly longer during sinus rhythm than during ventricular tachycardia or ventricular fibrillation. ${ }^{15}$

A cardiac cycle was defined to be a sequence of sample points extending from the midpoint be- 
tween two ventricular depolarizations to the next sequential midpoint. The total peak-to-peak amplitude of each cardiac cycle was divided into 30 equivalent amplitude subranges. Each sample point from the cardiac cycle whose amplitude fell within one of the subranges was counted and later plotted on an amplitude histogram. The isoelectric baselines of sinus rhythm and ventricular tachycardia were defined as the five amplitude subranges within a cardiac cycle which centered on the subrange with the greatest number of sample points. Normalization for differing heart rates was achieved by dividing the total number of sample points in each subrange by the total number of sample points in each cardiac cycle.

Ten second passages of sinus rhythm and ventricular tachycardia were compared using the assumption of amplitude distribution analysis (probability density function) that a significantly greater percentage of sample points occur on, or near, the isoelectric baseline during sinus rhythm than during ventricular tachycardia.

\section{Spectral Analysis}

Power spectral analysis was applied to a typical ventricular depolarization during sinus rhythm passage and a typical ventricular depolarization during ventricular tachycardia in each of the paired data sets. The mean was subtracted from the ventricular depolarization to eliminate DC offset. Templates were smoothed using a Hamming window to avoid the introduction of spurious high frequency artifact which results in abrupt truncation of the data with rectangular windows. ${ }^{24}$ The smoothed templates were padded with zeroes to form 1024-point data arrays to enhance resolution. A digital Fourier transform was then performed. The 1024-point power spectrum of each ventricular depolarization waveform pattern was calculated with a resultant frequency resolution of $0.98 \mathrm{~Hz}$.

Three classification techniques utilizing spectral analysis were examined. The first classification technique of spectral analysis examined the peak frequency component with the highest power as one feature to distinguish sinus rhythm from ventricular tachycardia. This classification scheme was based on the premise that ventricular depolarization during sinus rhythm would exhibit faster conduction with higher frequency components than ventricular depolarization during ventricular tachycardia. The second classification technique of spectral analysis examined the magnitude of the bandwidth having $3 \mathrm{~dB}$ points as limits. This classification scheme was based on an assumption that one of the two rhythms might have a wider spectrum of frequencies than the other. The third classification technique of spectral analysis compared the cutoff frequency during sinus rhythm versus ventricular tachycardia, defined as the frequency limit below which $95 \%$ of total power is contained. This technique was based on the assumption that one of the two rhythms might have more power over a wider range of frequencies than the other.

\section{Results}

Results from the processing of the thirty paired data sets are presented for each of the three analysis methods. Table 1 summarizes patient characteristics and the accuracy of each of the three detection algorithms for patients with normal and abnormal surface electrocardiographic QRS conduction during sinus rhythm in the absence of antiarrhythmic drug therapy. Table II summarizes the data for patients receiving antiarrhythmic drug therapy.

\section{Correlation Waveform Analysis}

Correlation waveform analysis was successful in discriminating all ventricular depolarizations during ventricular tachycardia from both normal and abnormal ventricular conduction during sinus rhythm using a linear boundary placed between 0.855 and 0.874 with a sensitivity of $100 \%$ and specificity of $100 \%$ (Fig. 2). This method was also successful in discriminating ventricular tachycardia from sinus rhythm during antiarrhythmic drug therapy with amiodarone, flecainide, quinidine, and procainamide. The dramatic change of morphology of ventricular activation upon initiation of tachycardia was easily detected by correlation of the ventricular waveform with the sinus rhythm template. (Fig. 3)

\section{Amplitude Distribution Analysis}

Amplitude distribution analysis discriminated 15 of 30 episodes $(50.0 \%)$ of ventricular 
Table I.

Patient Characteristics and Results of Digital Analysis Techniques without Antiarrhythmic Drug Therapy

\begin{tabular}{|c|c|c|c|c|c|c|c|c|c|}
\hline \multirow[b]{3}{*}{ Patient } & \multicolumn{6}{|c|}{ Characteristics } & \multirow[b]{3}{*}{ CWA } & \multirow[b]{3}{*}{ ADA } & \multirow[b]{3}{*}{ SA } \\
\hline & \multirow[b]{2}{*}{$\begin{array}{l}\text { Age/ } \\
\text { Sex }\end{array}$} & \multirow[b]{2}{*}{$\begin{array}{c}\text { Heart } \\
\text { Disease }\end{array}$} & \multicolumn{2}{|c|}{ Sinus Rhythm } & \multicolumn{2}{|c|}{$\begin{array}{l}\text { Ventricular } \\
\text { Tachycardia }\end{array}$} & & & \\
\hline & & & $\begin{array}{c}\text { QRS } \\
\text { Morphology }\end{array}$ & CL (ms) & $\begin{array}{c}\text { ORS } \\
\text { Morphology }\end{array}$ & $C L$ (ms) & & & \\
\hline 1 & $72 / \mathrm{M}$ & CAD & Normal & 1134 & LBB-S & 190 & + & + & + \\
\hline 2 & $14 / F$ & None & Normal & 730 & LBB-S & 540 & + & - & - \\
\hline 3 & $52 / \mathrm{M}$ & CAD & Normal & 745 & LBB-S & 247 & + & + & + \\
\hline 4 & $61 / M$ & CAD & Normal & 853 & INDI-S & 325 & + & + & - \\
\hline 5 & $66 / M$ & CAD & Normal & 633 & RBB-S & 260 & + & + & + \\
\hline 6 & $65 / M$ & CAD & LBBB & 729 & RBB-S & 278 & + & + & + \\
\hline 7 & $82 / M$ & CAD & LBBB & 975 & RBB-S & 422 & + & - & + \\
\hline 8 & $63 / F$ & CAD & RBBB & 577 & LBB-I & 223 & + & + & + \\
\hline 9 & $63 / M$ & CAD & IVCD & 610 & RBB-I & 330 & + & - & + \\
\hline 10 & $83 / F$ & HTN & IVCD & 918 & LBB-S & 290 & + & + & + \\
\hline 11 & $81 / M$ & CAD & IVCD & 699 & RBB-S & 320 & + & - & + \\
\hline 12 & $75 / M$ & CAD & IVCD & 704 & RBB-S & 430 & + & - & - \\
\hline 13 & $77 / \mathrm{M}$ & CAD & IVCD & 980 & LBB-I & 478 & + & - & + \\
\hline 13 & & & IVCD & 791 & LBB-ISO & 350 & + & - & - \\
\hline
\end{tabular}

The results show that correlation waveform analysis can distinguish ventricular tachycardia for patients without antiarnythmic drug therapy with 100\% accuracy. The other two methods, amplitude distribution analysis and spectral analysis, cannot distinguish ventricular tachycardia from sinus mythm with $100 \%$ accuracy for all patients. Abbreviations: $t=$ the two rhythms can be separated with $100 \%$ accuracy; - = the two ihythms cannot be separated with $100 \%$ accuracy; $A D A=$ amplitude distribution analysis; $C A D=$ coronary artery disease; CWA = correlation coefficient waveform analysis; $C L=$ cycle length; $H T N=$ hypertension; $I=$ inferior axis; IND = indeterminant; Iso = isoelectric axis; IVCD = intraventricular conduction delay; LBB = left bundle branch morphology; LBBB = left bundle branch block; RBB = right bundle branch morphology; RBBB = right bundle branch block; $S$

= Superior axis; SA = spectral analysis (cutoff frequency).

tachycardia from normal sinus rhythm. It identified four/five episodes $(80.0 \%)$ of ventricular tachycardia in group I, three/nine episodes (33.3\%) of ventricular tachycardia in group II, three/six episodes $(50.0 \%)$ of ventricular tachycardia in group III, and five/ten episodes $(50.0 \%)$ of ventricular tachycardia in group IV. The classification scheme required that the entire passage of sinus rhythm had a baseline percentage in a range well separated from that in ventricular tachycardia. Passages in which an overlap of ranges occurred were classified as a failure to discriminate between sinus rhythm and ventricular tachycardia since no threshold existed which provided complete separation. (Fig. 4)

\section{Spectral Analysis}

Using peak frequency to differentiate sinus rhythm from ventricular tachycardia, the peak frequency of sinus rhythm was greater than the peak frequency of ventricular tachycardia in 11 of 30 paired data sets $(36.7 \%)$, was the same as the peak frequency of ventricular tachycardia in 9 of: 30 paired data sets $(30.0 \%)$, and was less than the peak frequency of ventricular tachycardia in 10 of 30 paired data sets $(33.3 \%)$. Using the magnitude of the bandwidth with $3 \mathrm{~dB}$ points as limits, successful discrimination between sinus rhythm and ventricular tachycardia was possible in 17 of 30 episodes $(56.7 \%)$. Comparison of the cutoff fre- 
Table II.

Patient Characteristics and Results of Digital Analysis Techniques with Antiarnythmic Drug Therapy

\begin{tabular}{|c|c|c|c|c|c|c|c|c|c|c|c|}
\hline \multirow[b]{3}{*}{ Patient } & \multicolumn{8}{|c|}{ Characteristics } & \multirow[b]{3}{*}{ CWA } & \multirow[b]{3}{*}{ ADA } & \multirow[b]{3}{*}{ SA } \\
\hline & \multirow[b]{2}{*}{$\begin{array}{l}\text { Age/ } \\
\text { Sex }\end{array}$} & \multirow[b]{2}{*}{$\begin{array}{c}\text { Heart } \\
\text { Disease }\end{array}$} & \multirow[b]{2}{*}{$\begin{array}{c}\text { Antiarrhythmic } \\
\text { Therapy }\end{array}$} & \multirow{2}{*}{$\begin{array}{c}\text { Serum } \\
\text { Concentration } \\
(\mu \mathrm{g} / \mathrm{ml})\end{array}$} & \multicolumn{2}{|c|}{$\begin{array}{c}\text { Sinus } \\
\text { Rhythm }\end{array}$} & \multicolumn{2}{|c|}{$\begin{array}{l}\text { Ventricular } \\
\text { Tachycardia }\end{array}$} & & & \\
\hline & & & & & $\begin{array}{c}\text { QRS } \\
\text { Morphology }\end{array}$ & $\begin{array}{c}\mathrm{CL} \\
\text { (ms) }\end{array}$ & $\begin{array}{c}\text { QRS } \\
\text { Morphology }\end{array}$ & $\begin{array}{c}\mathrm{CL} \\
(\mathrm{ms})\end{array}$ & & & \\
\hline 4 & & & PROC & 5.7 & Normal & 819 & IND-S & 331 & + & + & - \\
\hline 5 & & & PROC & 5.0 & Normal & 580 & RBB-S & 505 & + & + & - \\
\hline 14 & $83 / F$ & $C A D$ & AMIO & 2.1 & Normal & 760 & LBB-Iso & 215 & + & + & - \\
\hline 15 & $75 / F$ & CAD & AMIO & 2.6 & Normal & 940 & RBB-I & 340 & + & - & + \\
\hline 15 & & & AMIO, PROC & $2.6,4.9$ & Normal & 945 & RBB-I & 420 & + & - & + \\
\hline 16 & $62 / \mathrm{M}$ & CAD & PROC & 5.5 & Normal & 540 & RBB-I & 200 & + & - & - \\
\hline 9 & & & QUIN & 2.5 & IVCD & 560 & RBB-I & 366 & + & + & + \\
\hline 10 & & & PROC & 12.6 & IVCD & 945 & RBB-S & 336 & + & - & + \\
\hline 11 & & & QUIN & ND & IVCD & 667 & RBB-S & 420 & + & - & - \\
\hline 12 & & & PROC & 12.7 & IVCD & 770 & RBB-S & 497 & + & + & - \\
\hline 12 & & & AMIO, FLEC & 1.6, ND & IVCD & 930 & RBB-I & 488 & + & - & + \\
\hline 15 & & & AMIO & 2.3 & RBBB & 845 & RBB-1 & 340 & + & - & - \\
\hline 15 & & & AMIO, PROC & 2.3, ND & RBBB & 945 & RBB-I & 380 & + & - & - \\
\hline 17 & $64 / M$ & CAD & AMIO & 2.1 & RBBB & 732 & IND-S & 366 & + & + & + \\
\hline 18 & $67 / M$ & VHD & AMIO & 2.2 & IVCD & 984 & LBB-S & 396 & + & + & + \\
\hline 18 & & & AMIO, PROC & $2.2,5.9$ & IVCD & 952 & RBB-I & 440 & + & + & + \\
\hline
\end{tabular}

The results show that correlation waveform analysis can distinguish ventricular tachycardia for patients with normal or abnormal conduction systems with antiarrhythmic drug therapy with $100 \%$ accuracy. Abbreviations: AMIO = amiodarone; FLEC = flecainide; ND = not determined; PROC = procainamide; QUIN = quinidine; VHP = valvular heart disease. The remaining abbreviations are the same as those of Table $I$.

quency during sinus rhythm versus ventricular tachycardia was the most successful of the three spectral analysis techniques in identifying ventricular tachycardia (18 of 30 episodes, $60.0 \%)$. It identified three of five episodes $(60.0 \%)$ of ventricular tachycardia in group I, seven of nine episodes $(77.8 \%)$ of ventricular tachycardia in group II, two of six episodes (33.3\%) of ventricular tachycardia in group III, and six of ten episodes $(60.0 \%)$ of ventricular tachycardia in group IV. Figure 5 shows two examples of power spectral plots of ventricular depolarization during sinus rhythm and ventricular tachycardia in the same patient, with and without antiarrhythmic drug therapy.

\section{Discussion}

Using correlation waveform analysis, a single discriminant function was successful in distin- guishing ventricular tachycardia from sinus rhythm with $100 \%$ accuracy in patients with normal or aberrant surface electrocardiographic QRS conduction during sinus rhythm, in the absence and presence of antiarrhythmic drug therapy. In contrast, $15 / 30$ episodes $(50 \%)$ of ventricular tachycardia were identified using amplitude distribution analysis, and a maximum of $18 / 30 \mathrm{epi}-$ sodes $(60 \%)$ of ventricular tachycardia were identified using spectral analysis with Fast Fourier transformation. The correlation waveform method has been associated with some modest computational complexity but is capable of execution in real time ${ }^{18}$ making it feasible for use in an implantable antitachycardia device.

Antitachycardia devices currently available utilize a preprogrammed minimum rate as the essential means of detecting the onset of a pathologic tachycardia. ${ }^{1-5}$ However, complications may occur when increases in the sinus rate or parox- 


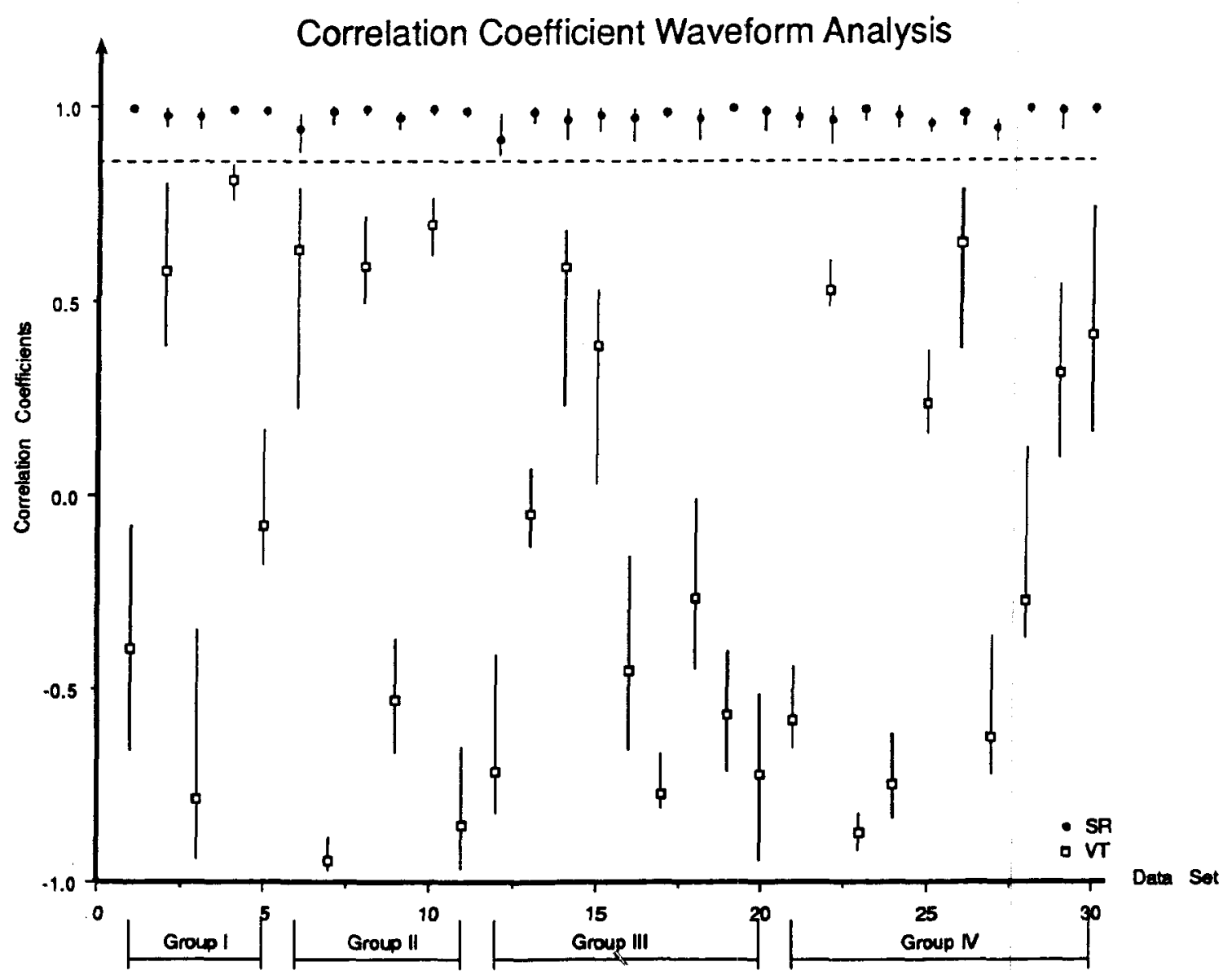

Figure 2. Results of correlation waveform analysis of the 30 paired data sets of sinus rhythm (SR) and ventricular tachycardia (VT). Solid circles are average values and vertical bars represent the range of correlation coefficients for each patient during sinus rhythm. Open squares are average values and the vertical bars represent the range of correlation coefficients during ventricular tachycardia. Each paired set is aligned vertically. The dotted line represents a decision boundary (between 0.855 and 0.874 ) which separates sinus rhythm from ventricular tachycardia with $100 \%$ accuracy. Groups I-IV are defined in the text.

ysmal occurrences of other arrhythmias, which exceed the preprogrammed minimum detection rate, are misdiagnosed as the pathological tachycardia and result in programmed stimulation and possible initiation of a pathological tachycardia by the tachycardia-terminating device. ${ }^{14}$ In other cases, a spontaneous occurrence of ventricular tachycardia may fail to be detected and treated by the tachycardia-terminating device because the rate of the ventricular tachycardia is slower than the preprogrammed minimum detection rate. ${ }^{15}$ Other commercially available tachycardia-terminating devices have utilized additional rate crite. ria such as analysis of onset or sustained high rate. ${ }^{1,2}$ These criteria have not proven to be uniformly reliable, especially when the pathological tachycardia occurs paroxysmally during sinus tachycardia, or when sinus tachycardia follows termination of the pathological tachycardia by the tachycardia-terminating device.

The implantable cardioverter defibrillator: designed by Mirowski has utilized the combina. tion of amplitude distribution analysis (probability density function) with a minimum detection rate for the detection of ventricular fibrillation. This method utilizes the striking absence of a 
PANEL A
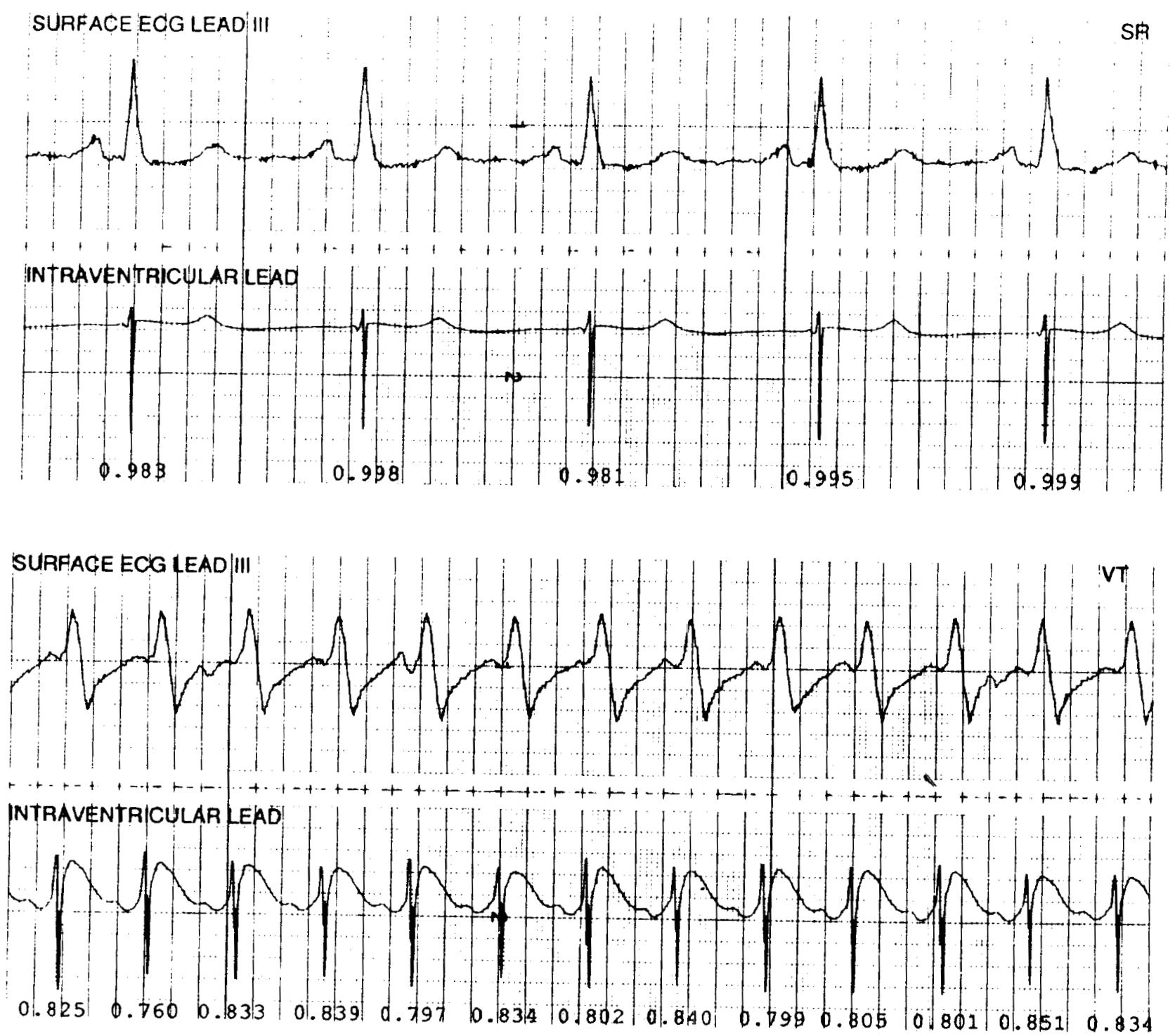

Figure 3. Ventricular electrograms during sinus rhythm (top) and ventricular tachycardia (bottom) in two patients. Panel A is from a patient in Group I (normal conduction during sinus rhythm without drugs) and Panel B is from a patient in group II (intraventricular conduction delay during sinus rhythm without drugs). Differences in waveform morphology, when ventricular tachycardia is compared to sinus rhythm, are apparent from the tracings. Correlation coefficients are given below each ventricular depolarization. Chart speed is $50 \mathrm{~mm} / \mathrm{sec}$.

peak at amplitude zero in the differentiated signal as a means of distinguishing ventricular fibrillation from other arrhythmias and sinus rhythm. However, this combination of detection criteria has had limited applicability for patients having recurrent ventricular tachycardia as well as re- current ventricular fibrillation. In many cases, the activity peak of ventricular tachycardia may occur at amplitude zero, resulting in failure to detect the presence of ventricular tachycardia by probability density function. ${ }^{10}$

In the present study amplitude distribution 
PANEL B
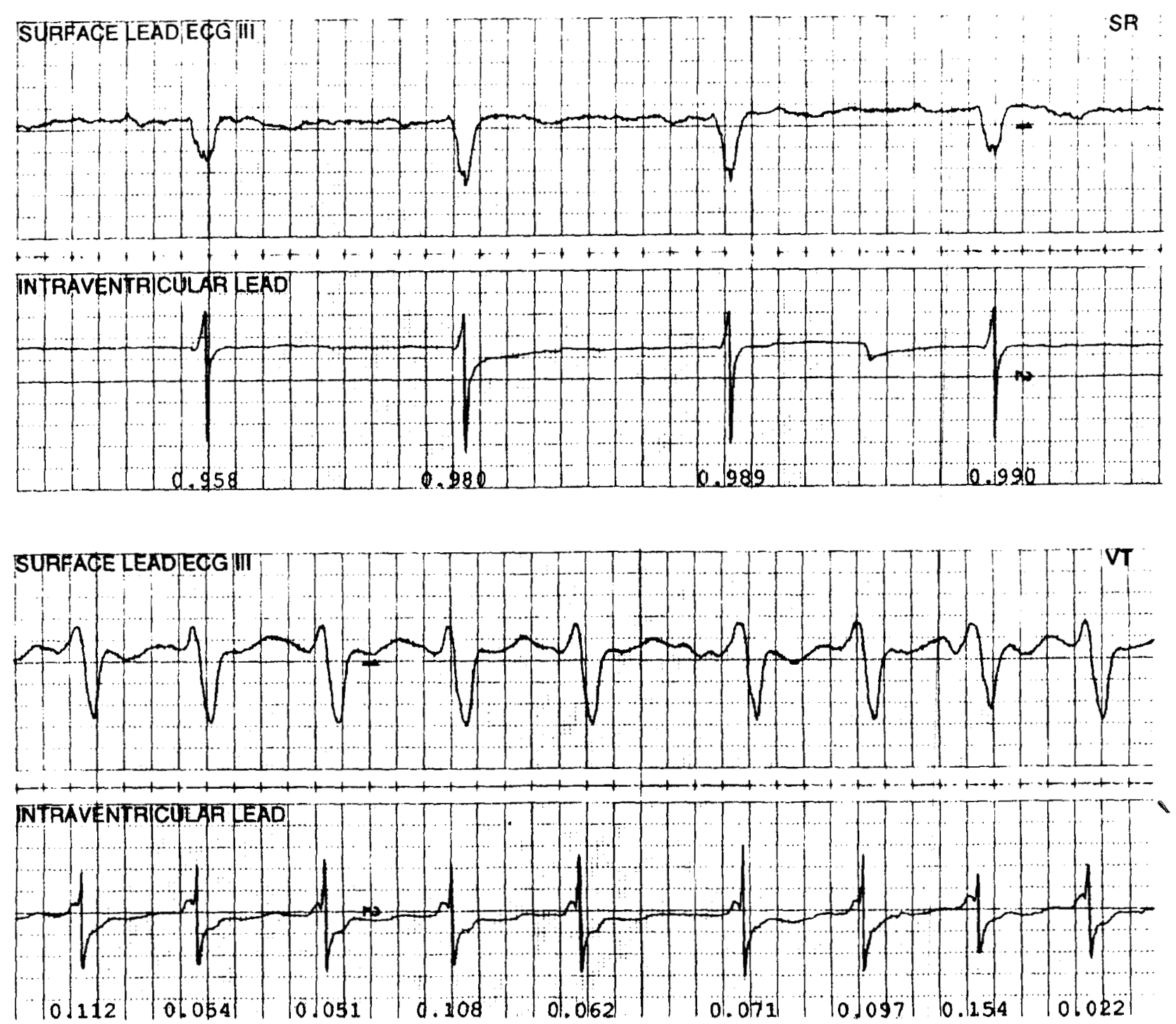

Figure 3b. Continued.

analysis was found to be effective in the identification of ventricular tachycardia for the patients with normally conducted sinus rhythm without antiarrhythmic drug therapy, but not for the patients with an abnormal conduction system, especially those with intraventricular conduction delay, bundle branch block, and antiarrhythmic drug therapy. Changes in amplitude distribution as a result of an intraventricular conduction delay, bundle branch block, or antiarrhythmic drug therapy appears to have resulted in a smaller percentage of time spent on or near the baseline during sinus rhythm when compared to ventricular tachycardia in these groups (Tables I and II). Since performance in patients with normal ventricular conduction during sinus rhythm was acceptable, and the time required to calculate the distribution was less than that required to calculate a correlation coefficient for a similar cycle, amplitude distribution analysis may provide a 
LIN, ET AL.

PANEL A
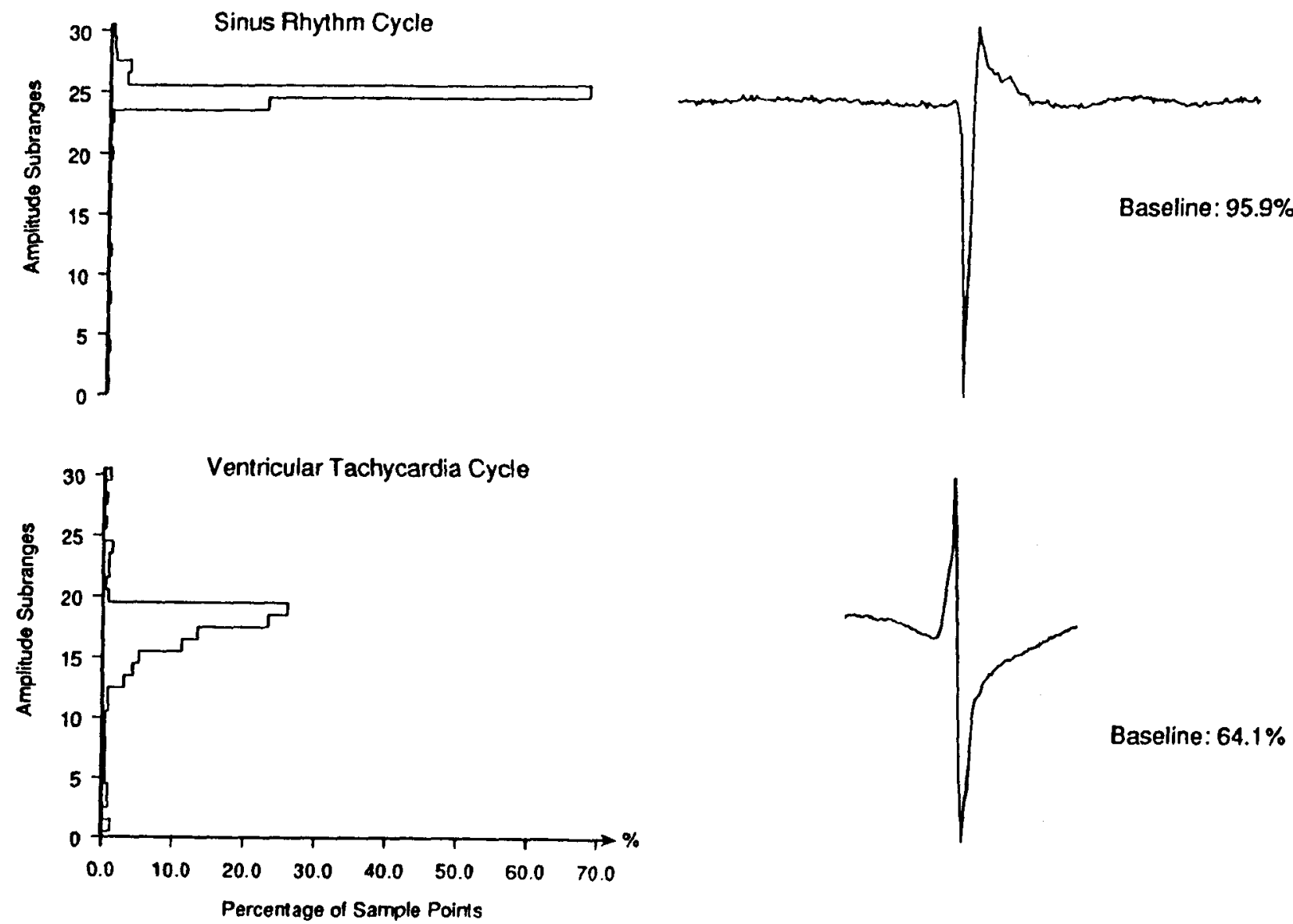

Baseline: $64.1 \%$

Figure 4. Examples of success (Panel A) and failure (Panel B) in identifying ventricular tachycardia by amplitude distribution analysis in individual patients. An amplitude histogram of a cardiac cycle with its associated ventricular electrogram during sinus rhythm is shown in the upper half, and an amplitude histogram of a cardiac cycle during ventricular tachycardia with its associated ventricular electrogram during subsequently induced ventricular tachycardia is shown in the lower half of each panel. The peak-to-peak amplitude range of the cardiac cycle has been divided into 30 subranges and temporal normalization was performed by dividing by the number of sample points in each subrange by the total number of sample points within the cardiac cycle. The percentage of sample points falling within each subrange has then been plotted on a histogram. Determination of the baseline percentage of sample points on, or near, the isoelectric baseline has been made from the 5 subranges centered on the most populated subrange. During sinus rhythm, $95.9 \%$ of all amplitude points fall within the baseline region while only $64.1 \%$ fall within the baseline region during ventricular tachycardia. In Panel A (patient 8), the baseline percentage of sample points during ventricular tachycardia was significantly less than the baseline percentage during sinus rhythm, thus successfully identifying ventricular tachycardia. In Panel B (patient 10 during antiarrhythmic drug therapy), the opposite occurred, preventing successful detection of ventricular tachycardia by amplitude distribution analysis. 
PANEL B
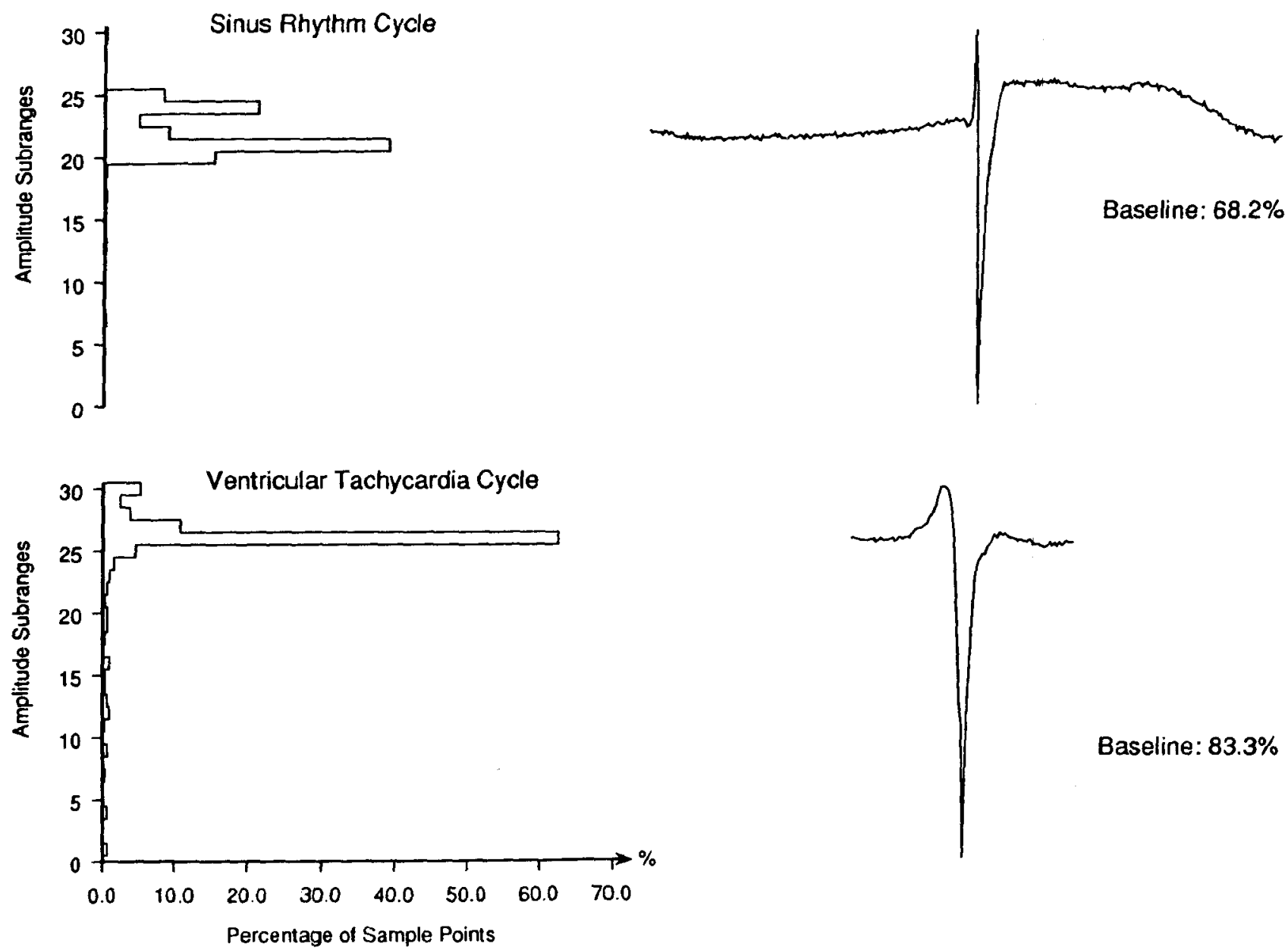

Baseline: $\mathbf{8 3 . 3 \%}$

Figure 4b. Continued.

valid alternative for the patients with a normal conduction system when speed and simplicity are essential.

None of the three techniques of spectral analysis using Fast Fourier transformation produced a distinct discrimination function suitable for all groups of patients. Because ventricular depolarization using the His-Purkinje system during sinus rhythm is faster than ventricular depolarization during ventricular tachycardia, more high frequency components with higher powers were anticipated to be seen in sinus rhythm than in ventricular tachycardia. However, the results suggest that this may not be always true, whether or not there may be abnormal QRS conduction during sinus rhythm.

\section{Limitations}

Our investigation was an acute study which utilized temporary implantation of electrode catheters having $1 \mathrm{~cm}$ interelectrode spacing. The results of a similar investigation may or may not differ with chronically implanted electrode catheters having similar, wider, or more narrow interelectrode spacing.

Our investigation demonstrated correlation waveform analysis to be consistently reliable in 
PANEL A

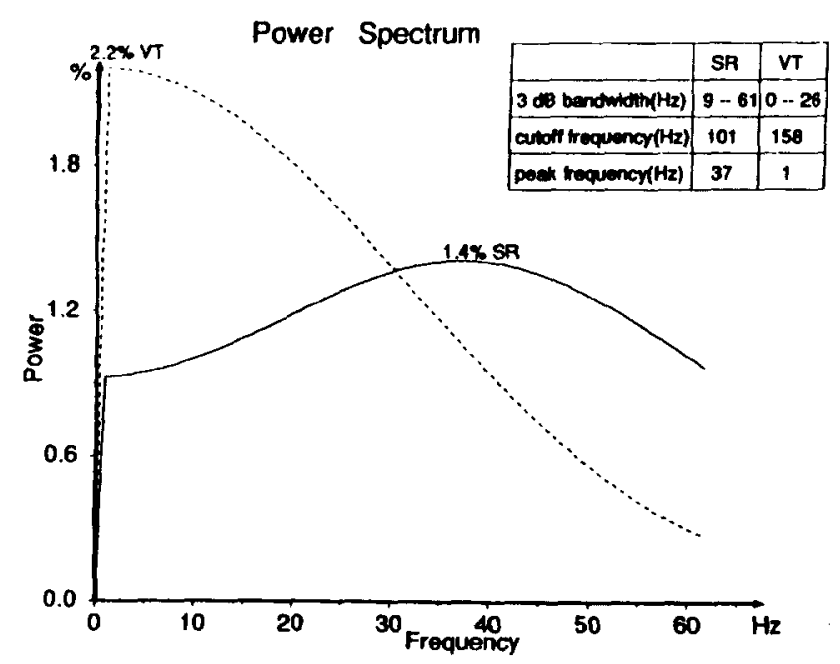

PANEL B

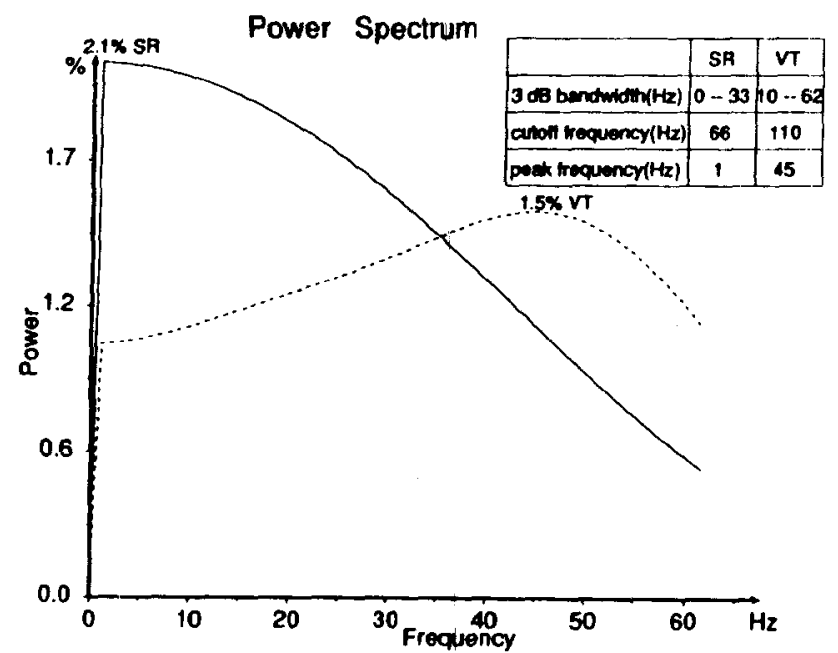

Figure 5. Typical power spectrum plots of ventricular depolarization during sinus rhythm and ventricular tachycardia from one patient (patient 4) without antiarrhythmic drug therapy (Panel A) and with antiarrhythmic drug therapy (Panel B). The peak frequency content of the depolarization waveform from each of the two rhythms has been plotted as a percent of the power. Identifcation of ventricular tachycardia has been based on the hypothesis that slower conduction (i.e. widened waveforms) during ventricular tachycardia result in a shift of peak frequency to a lower value than that observed during sinus rhythm. However, whereas sinus rhythm has a higher peak frequency than ventricular tachycardia in Panel $A$, the converse is observed in Panel B. No consistent pattern is present in the differences in bandwidths having $3 \mathrm{~dB}$ points as limits, or in the cutoff frequency, to distinguish sinus rhythm from ventricular tachycardia.

distinguishing ventricular from sinus rhythm in the presence of several antiarrhythmic drugs. Because our investigation utilized acute electrode catheter placement, we were not able to determine whether antiarrhythmic drugs might change the ventricular electrogram template observed during sinus rhythm. Should the template of ventricular activation during sinus rhythm need to be reestablished after administration of antiarrhythmic drugs, an adaptive template method such as is used in surface electrocardiography ${ }^{25}$ may be appropriate.

There were only two patients in this study whose analysis included ventricular tachycardia with a right bundle branch morphology being compared to sinus rhythm with right bundle branch block (Tables I and II). There were no patients in this study whose analysis included ventricular tachycardia with a left bundle branch morphology being compared to sinus rhythm with left bundle branch block. Larger studies incorporating more patients with similar bundle branch morphologies during ventricular tachycardia and sinus rhythm may be necessary to determine whether correlation waveform analysis remains consistently reliable in distinguishing ventricular tachycardia from sinus rhythm in these two subsets of patients.

\section{Further Study and Future Improvement of Ventricular Tachycardia Diagnosis By Antitachycardic Devices}

It remains to be determined whether correlation waveform analysis will be helpful in distinguishing ventricular tachycardia from other tachycardias in the subset of patients who develop aberrant ventricular depolarization during sinus or other, supraventricular tachycardias. Nonetheless, the establishment of a reliable means of detecting real-time changes in ventricular electrogram morphology by the present study provides an important first step toward the goal of improved tachycardia diagnosis by an antitachy- 
cardia device. Preliminary work suggests that reliable, real-time, beat-to-beat analysis of cardiac rhythms may be possible when based upon correlation waveform analysis of atrial and ventricular depolarizations, determination of the time intervals between successive atrial depolarizations and ventricular depolarizations, respectively, and determination of the time interval between successive atrial and ventricular depolarizations. ${ }^{26,27}$

\section{Summary}

Correlation waveform analysis appears to be a reliable technique for distinguishing ventricular

\section{References}

1. Sowton E. Clinical results with the tachylog antitachycardia pacemaker. PACE 1984; 7:1313-1317.

2. Charos GS, Haffajee CI, Gold RL, et al. A theoretically and practically more effective method for interruption of ventricular tachycardia: Selfadapting autodecremental overdrive pacing. Circ 1986; 73:2:309-315.

3. Reddy CP, Todd EP, Kuo CS, et al. Treatment of ventricular tachycardia using an automatic scanning extrastimulus pacemaker. J Am Coll Cardiol 1984; 3:1:225-230.

4. Spurrell RAJ, Nathan AW, Camm AJ. Clinical experience with implantable scanning tachycardia reversion pacemakers. PACE 1984; 7:1296-1300.

5. Higgins JR, Swartz JG, Dehmer GJ, et al. Automatic scanning extrastimulus pacemaker to treat ventricular tachycardia. PACE 1985; 8:101-109.

6. Olson WH, Bardy GH, Mehra R, et al. Tachyarrhythmia detection algorithm for an implantable cardioverter and defibrillator. (abstract) Circulation 1986; 74:II-110.

7. Bella PD, Brugada P, Dugernier T, et al. Transventricular lead system for identification and termination of tachycardias. (abstract) Circulation 1986; 74:II-183.

8. Davies DW, Wainwright RJ, Tooley MA, et al. Detection of pathological tachycardia by analysis of electrogram morphology. PACE 1986; 9:200-208.

9. Davies DW, Wainwright RJ, Tooley MA, et al. Endocardial electrogram analysis for the automatic recognition of ventricular tachycardia. (abstract) Circulation 1985; 72:III-474.

10. Mirowski M, Mower MM, Reid PR, et al. The Automatic Implantable Defibrillator, New modality for treatment of life-threatening ventricular arrhythmias. PACE 1982; 384-401.

11. Langberg JJ, Griffin JC. Arrhythmia identification using the morphology of the endocardial electrogram. (abstract) Circulation 1985; 72:IIl:474. tachycardia from sinus rhythm using intracavitary ventricular electrograms. Its computational demands are modest, making it suitable for consideration of its incorporation in tachycardia diagnosis by an implantablei antitachycardia device.

Acknowledgments: The authors wish to express their appreciation to Kevin Price, B. S., and Ryan Reeg, B. S., for their technical assistance in the cardiac electrophysiology laboratory, and Ms. Debbie Laird for preparation of this manuscript.

12. Griffin JC, Nielsen AP, Finke WL, et al, A new method of rhythm identification: Endocardial electrogram morphology. (abstract) Circulation 1984; 70:II-201.

13. Mercando A, Gabry M, Klementowicz P, et al. Detection of ectopy by measurement of ventricular activation sequence using two electrodes. (abstract) J Am Coll Cardiol 1986; 7:2:184A.

14. Fahraeus T, Lassvik C, Sonnhag C. Tachycardias initiated by automatic antitachycardia pacemakers. PACE 1984; 7:1049-1054.

15. Mirowski M, Reid PR, Mower MM, et al. Clinical performance of the implantable cardioverter-defibrillator. PACE 1984; 7:1345-1350.

16. Feldman CL, Amazeen PG, Klein MD, et al. Computer detection of ventricular ectopic beats. Comp Biomed Res 1970; 3:666-674.

17. Arzbaecher R, Biancalana P, Stibolt T, et al. Computer technique for detedtion of cardiac arrhythmias. J Assoc Adv Med lnstr 1971; 5:104.

18. Jenkins JM, Wu D, Arzbaecher RC. Computer diagnosis of supraventricular and ventricular arrhythmias. Circulation 1979; 60:977-987.

19. Lin D, Jenkins JM, DiCarlo LA. Analysis of P-wave changes on the esophageal electrogram: A twolead arrhythmia analysis system using morphology and timing of both atrial and ventricular activation. Comp Cardiol 1987 (In press).

20. Lin D, Jenkins JW, Wiesmeyer MD, et al. Analysis of time and frequency domain patterns of endocardial electrograms to distinguish ventricular tachycardia from sinus rhythm. Comp Cardiol 1986; 171-174.

21. Morady F, Dicarlo L, Winston S, et al. A prospective comparison of triple extrastimuli and left ventricular stimulation in studies of ventricular tachycardia induction. Circulation 1984; 70:1:5257.

22. Marriott HJL. Bundle branch block. In: Practical 
LIN, ET AL.

Electrocardiography. Seventh edition. Baltimore/ London; Williams \& Wilkins, 1983 p. 62-83.

23. Blum JR, Rosenblatt JI. Probability and Statistics. Philadelphia, W.B. Saunders Co., 1972 p. 193.

24. Oppenhiem AV, Schafer RW. Digital Signal Processing. Englewood Cliffs, NJ, Prentice-Hall Inc. 1975 p. 242.

25. Hsia PW, Jenkins JM, Shimoni Y, et al. An automated system for ST segment and arrhythmia analysis in exercise radionuclide ventriculogra. phy. IEEE Trans Biomed Eng 1986; 33:6:585-593.

26. Arzbaecher R, Bump T, Jenkins JM, et al. Automatic tachycardia recognition, PACE 1984; 7:5415.77.

27. Jenkins JM, Bump T, Munkenbeck F, et al. Tachycardia detection in implantable antitachycardia devices, PACE 1984; 7:1273-1277. 